\title{
AMELIORATION OF MACHINE LEARNING AND ARTIFICIAL INTELLIGENCE IN MEDICAL MANAGEMENT (A CASE STUDY ON THE PANDEMIC OF COVID-19 CASES IN ASIA)
}

\author{
Sagaya Aurelia ${ }^{1}$, Felcy Judith ${ }^{2}$ \\ ${ }^{1}$ Assistant Professor, CHRIST University, Bangalore \\ Sagaya.aurelia@christunversity.in \\ ${ }^{2}$ Director (MCA) and Associate Professor. John College, Bangalore, India \\ felcy_judith@yahoo.com
}

\begin{abstract}
:
Machine learning is seeing increasingly utilized in medical management for different reasons: tremendous sums of information are being captured and made accessible carefully; handling of vast sums of information has gotten to be cost-effective due to the expanded computing control presently accessible at reasonable costs; and different open source systems, toolkits, and libraries are accessible that can be utilized to construct and execute ML applications. Particularly in healthcare, ML has driven to energizing modern improvements that may rethink COVID-19 treatment and vaccination within a long time to come. With modern occurrences of the brand modern coronavirus clutter, COVID-19, creating day through the day, it is common to compare the unused affliction to other flare-ups in current history. Machine learning can offer assistance to anticipate three sorts of restorative dangers - disease, seriousness, and result. Whereas it is still it is early for COVID-19 with machine learning, but early applications seem promising. Machine learning is utilized when a computer has been instructed to recognize designs by giving it with information and a calculation to assist get it that information. This method of learning from the information is called training and the yield that we accomplish is through testing. Machine learning-based robotic surgery is
\end{abstract}

changing the way surgery is performed nowadays. Machine learning in healthcare is getting to be more broadly utilized and is making a difference in patients and clinicians in numerous diverse ways. Cybersecurity and protection are major concerns and open challenges in healthcare which are yet to be addressed.

Keywords:Machine learning, AI, pandemic, COVID-19, health care, Asia, prediction analysis

\section{INTRODUCTION}

Machine learning (ML) is an apposite of Artificial intelligence (AI) wherein the framework looks at perceptions of information, such as cases, coordinate encounter, or instruction and figures out designs in information and predicts occasions within the future based on the cases that we offer. The process of machine learning is shown in fig. 1 . Machine learning is seeing increasingly utilize over businesses for different reasons: tremendous sums of information are being captured and made accessible carefully; handling of huge sums of information has gotten to be cost-effective due to the expanded computing control presently accessible at reasonable costs; and different open source systems, toolkits, and libraries are accessible that can be utilized to construct and execute ML applications. 
Particularly in healthcare, ML has driven to energizing modern improvements that may rethink COVID-19 treatment and vaccination. ML can increase to get treatment in creating nations which don't have sufficient master specialists that can treat certain maladies, it can progress the affectability of discovery, include more esteem in treatment choices, and it can offer assistance in personalizing treatment so that each one gets the treatment that's best for them. In numerous cases, they can indeed include workflow proficiency in clinics. The conceivable outcomes are perpetual [6$10]$.

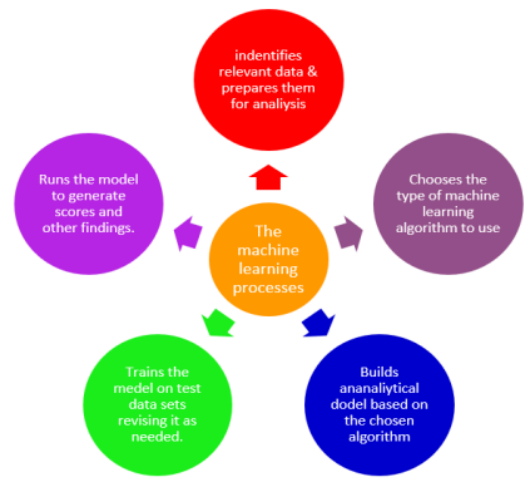

Fig. 1: Machine Learning Process

\section{PANDEMICS IN THE PAST}

With modern occurrences of the brand modern coronavirus clutter, COVID-19, creating day through the day, it is common to compare the unused affliction to other flare-ups in current history. There turned into the $1918 \mathrm{flu}$, for the case, that tainted nearly a third trusted Source of the world's populace prior to it failed out. At that point got here other undermining infections that respected out of no place: SARS, H1N1 flu in 2009, and Ebola. By the by, the aftermath of each affliction to a great extent depends upon diverse circumstances - when we capture it, how infectious and dangerous it is, how to clean individuals are, and how quickly an antibiotic or remedy is available. Fig. 2 and Fig.3 depict the affected and death cases due to various pandemics in the past.

The 1918 Spanish flu plague was the deadliest flu season we know of, tainting around one-third of the world's population. The 1918 widespread strain of flu was unused and novel for most individuals were between 40 or 50 years old, but that is where the passing rate indeed was high. Back at that point, researchers did not know infections caused illness, and however, we did not have an antibody or antiviral to assist in anticipating or treating flu, nor did we have antimicrobial to treat auxiliary bacterial diseases.

SARS is another sort of coronavirus that came out of China and spread rapidly through respiratory beads. In spite of the fact that the SARS passing rate was higher than COVID-19, COVID19 has, as of now, claimed more lives. By and large, in spite of the fact that SARS' passing rate was high, COVID-19 has driven to more fatalities, more financial repercussions, and more social repercussions than with SARS [19].

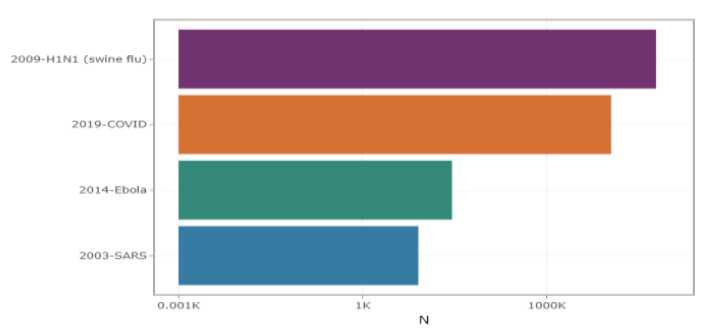

Fig. 2: Affected Cases Due to Various

\section{Pandemics in the Past}

Back in 2009, an unused sort of flu an H1N1 strain popped up, and individuals froze since we didn't have an antibody, and the novel strain was spreading fast. Like COVID-19, there was no insusceptibility at the beginning of the episode. We did have antiviral to encourage recuperation, and by the end of 2009, we had an antibiotic which combined with higher levels of resistance would give assurance in future flu seasons. Still, it claimed over 12,000 lives within the USA. 
Ebola was dangerous, killing up to 50 percent of those who got wiped out. But since it transcendently spread through real liquids like sweat and blood amid the final stages of the malady, it wasn't as infectious as COVID-19. Additionally, since side effects were so serious, wellbeing authorities were able to rapidly distinguish those who were in proximity with individuals and disconnect them [18].

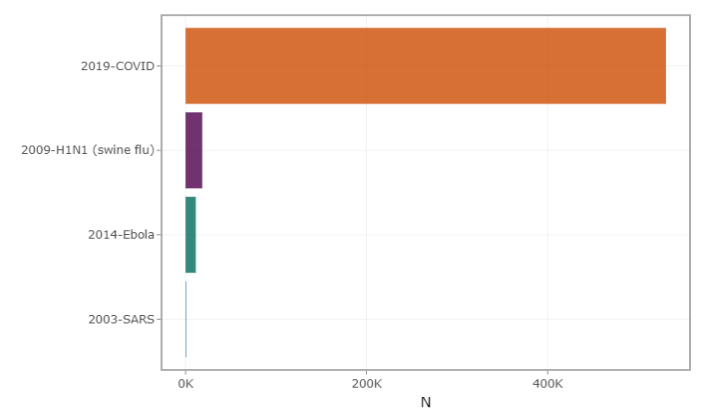

Fig. 3: Death Rate Due to Various Pandemics in the Past

\section{ML IN COVID-19}

The part of AI and Machine Learning in today's world cannot be thought little of nor ignored. In reality, this region of science has gotten to be vital to the times we live in. Seeing at the current scenario - viral pandemics are a genuine danger. COVID-19 is not the primary, and it will not be the final. The contrast is, nowadays, hundreds of inquire about groups around the world are collecting and sharing information to create arrangements, and machine learning is making a difference in them do so. Researchers and investigators are utilizing ML[11-15]. Few of them are as follows.

\section{Identifying Disease and Diagnosis}

With developing populaces and expanded life hope, wellbeing frameworks as shown in Fig.4. are rapidly getting to be overburdened, underresourced, and not prepared for the challenges they confront. Researchers have been working on ML models that anticipate malady helplessness or help in the early stage of infections and sicknesses. UKbased innovation start-up Feebris is utilizing manufactured insights calculations for the exact location of complex respiratory conditions within the field. It interfaces to existing restorative sensors and can be utilized by non-doctor clients to recognize respiratory issues early, maintaining a strategic distance from complications and hospitalizations.

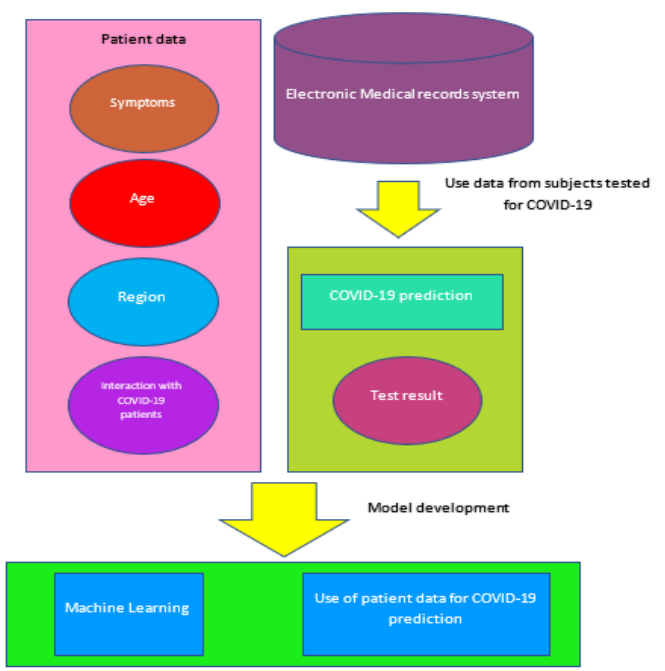

Fig. 4: Machine Learning-Based Covid-19 Identification Model

To say the slightest, Machine Learning is a vital tool in battling the current widespread. In a case in this opportunity to gather information, pool our information, and combine our competence, able to spare numerous lives - both presently and within the future.

\section{Churn Analysis}

The information collected from the patients who visited the specialists can offer assistance in producing valuable criticism for the long run era. Machine Learning fueled churn examination gives us the data on whether or not they will return to the same clinic for any kind of treatment in the future. Utilizing the verifiable information of the patients, able to anticipate the at-risk patients, who may not return to the same clinic for any future medications, 
by relegating them with a churn proportion and discover any openings to bring them back in future. Client lifetime esteem is the advantage that the health center makes from one specific individual.

Predicting the chance of contamination, machine learning has made a difference in deciding the hazard variables such as age, pre-existing conditions, universal cleanliness propensities, social propensities, etc. Outstandingly, Dave DeCaprio and others have utilized machine learning to construct a starting Helplessness File for COVID-19. Anticipation measures such as wearing veils, washing hands, and humane removal are all likely to impact general hazards.

\section{Predictive Analysis}

Predictive analysis is the auxiliary of progressed analytics that predicts approximately obscure future occasions. Its primary employments are information mining, insights, modeling, machine learning, and manufactured insights to analyze the current information and make forecasts of the up and coming slant and behavior. The design found in chronicled and value-based information can be utilized to distinguish dangers and openings within the future. Predictive analysis applications in wellbeing care can recognize patients who are at the chance of creating certain conditions or lifetime sicknesses. One of the foremost surprising breakthroughs, particularly for the healthcare segment, is Computer Vision combined with machine learning and artificial intelligence [20].

\section{a. Early Prediction Using Machine Learning}

In brief, machine learning is to memorize valuable data from a massive sum of information utilizing its algorithm shown for particular issues. Machine learning ranges an assortment of areas, such as medicine, computer science, measurements, building innovation, brain research, etc. For illustration, a neural network, a relatively develop machine learning calculation, can reenact any highdimensional non-linear optimal mapping between input and yield by copying the preparing work of the natural brain's nervous system. When confronted with complex information relations, the traditional measurable strategy is not such effective, which may not get precise about as the neural network[16,17].

Since most modern, irresistible illnesses happening in human creatures are of creature root, it is a successful prerequisite to foresee illnesses by deciding the common intrinsic characteristics of species and natural conditions that lead to the flood of modern diseases. By analyzing the inborn characteristics of wild species through machine learning, unused reservoirs and carriers of zoonotic maladies can be precisely predicted 19 . The general objective of the machine learning-based approach is to expand causal deduction hypothesis and machine learning to identify and evaluate the principal imperative components that cause zoonotic infection flare-ups and to produce visual tools to demonstrate the complex causal connections of irresistible creature illnesses and their relationship with zoonotic diseases 20 . In any case, the highly nonlinear and complex issues to be analyzed within the early prediction show of irresistible maladies based on machine learning more often than not lead.

\section{b. Prediction Model Selection:}

\section{i. Gompertz Models}

Gompertz model was initially proposed by Gomperts as being a populace growth model to portray the termination law of the populace. The improvement of irresistible illnesses is similar to the development of people and populaces. Gompertz model is chosen to depict the spread law of irresistible infections and to consider the 
components that control and influence the spread of COVID- 19.

$$
Q_{t}=a e^{-b e^{-c\left(t-t_{0}\right)}}
$$

Qt is the aggregate affirmed cases;

a is the anticipated greatest of affirmed cases.

$b$ and $c$ are fitting coefficients. $t$ is the number of days. $\mathrm{t} 0$ is the first case in occurrence time.

\section{Logical Model}

The logical model is utilized in the study of disease transmission. It is common to investigate the hazard variables of certain diseases and anticipate the likelihood of event of a particular infection agreeing to the hazard variables. The improvement and transmission law of the study of disease transmission can be anticipated through calculation as expression (2)

$$
Q_{t}=\frac{a}{1+e^{b-c\left(t-t_{0}\right)}}
$$

Qt is the total affirmed cases;

$\mathrm{a}$ is the anticipated most extreme of affirmed cases. $b$ and $c$ are fitting coefficients. $t$ is the number of days. t0 is the time when the first case occurred.

\section{Bertalanffy Model}

Bertalanffy's model is regularly utilized as a development model. It is basically utilized to consider the variables that control and affect development. It is utilized to portray the development characteristics of the angle. Moreover, other species can be used to depict the development of creatures, such as pigs, steeds, cattle, sheep, etc. and other irresistible diseases. The advancement of irresistible infections is comparable to the development of people and populaces. In this paper, Bertalanffy's model is chosen to portray the spread law of irresistible illnesses and to consider the factors that control and influence the spread of COVID-19.

$$
Q_{t}=a\left(1-e^{-b\left(t-t_{0)}\right.}\right)^{c}
$$

Qt is the aggregate affirmed cases;

a is the anticipated greatest of affirmed cases.

$b$ and $c$ are fitting coefficients. $t$ is the number of days.

t0 is the time when the first case occurred

\subsection{Asia - A Case Study}

The COVID-19 widespread started in Asia in Wuhan, Hubei, China, and has spread broadly through the landmass. As of 8 June 2020, at the slightest, one case of COVID-19 had been detailed in each nation in Asia but North Korea and Turkmenistan. Countries with the most elevated numbers of affirmed coronavirus cases are India, Iran, Turkey, Pakistan, and Saudi Arabia. Among the promptest nations to report COVID-19 cases after the flare-up in China were South Korea, Taiwan, and Vietnam, but these nations had effectively controlled the widespread. The most prominent numbers of active cases are recorded in Iran, India, Turkey, China, and Pakistan, with more than 27,000 cases combined as shown in fig. 8 . The cases tolls in several nations, in any case, are claimed to be altogether higher than those given in official.Fig.5,Fig 6, and Fig.7 depict the affected rate, geographical wise affected rate, and the death rate of Asia[1,2].

A few Southeast Asian nations experienced a critical rise in cases taking after a Tabligh Jamaat occasion from 27 February to 1 March. Walk at a mosque in Kuala Lumpur, where numerous individuals are accepted to have been contaminated. So also, occasions in India and Pakistan have too caused a surge within the number of cases in those nations. Major flare-ups developed in dormitories for vagrant specialists within the Maldives and Singapore, where social distancing was incapable of being practiced and 
driven to a critical rise in cases in both countries [21].

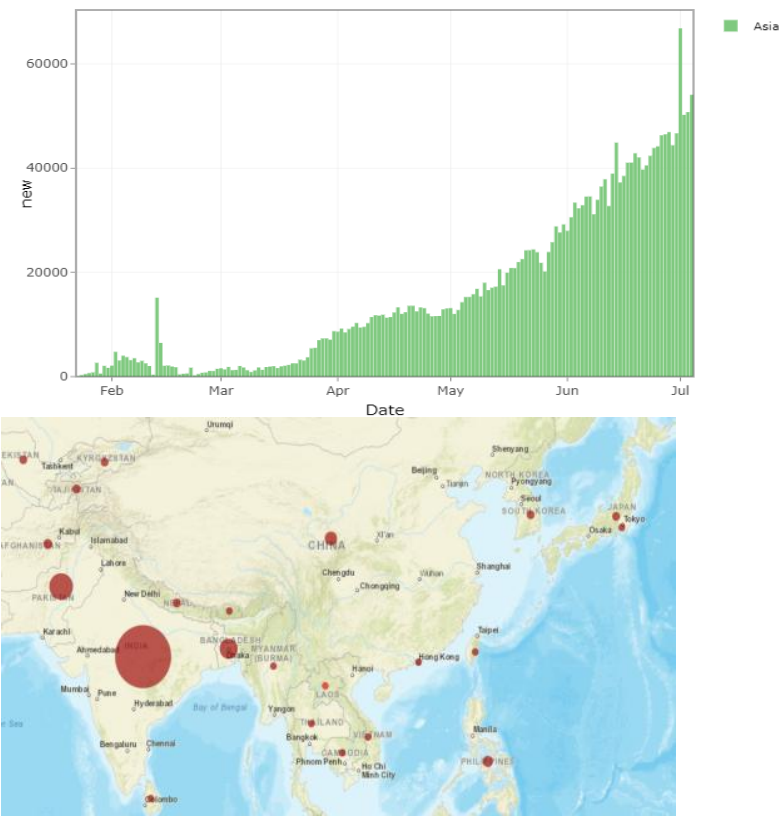

Fig. 5: No of Active Cases in AsiaFig. 6:

Geographical Wise Affected Places in Asia

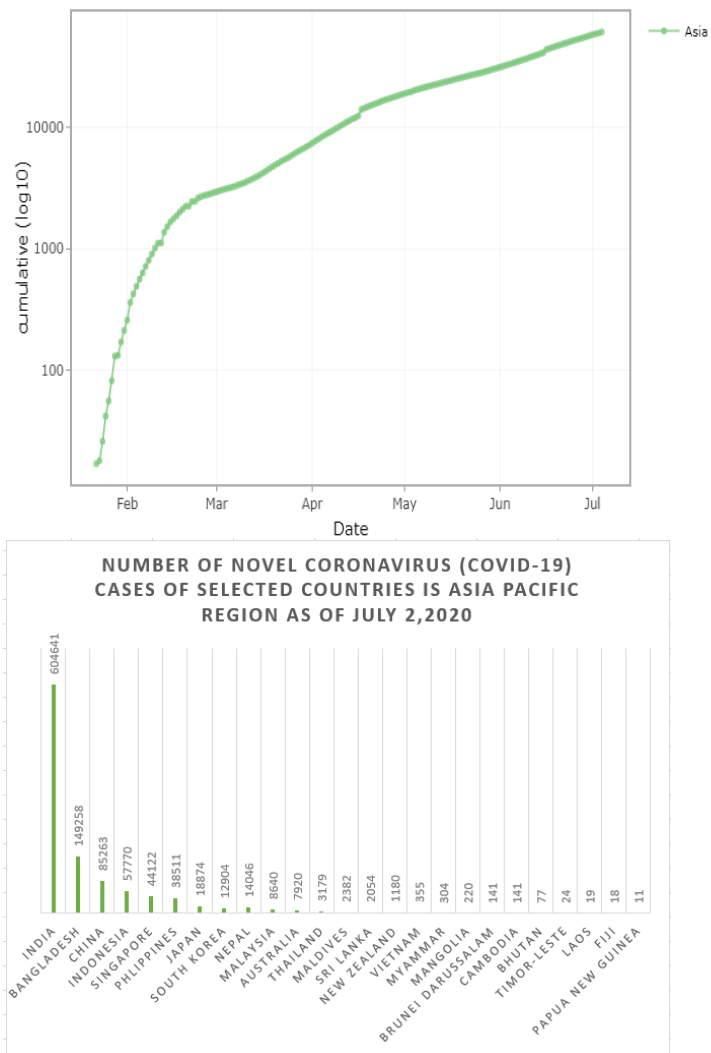

Fig. 7: Death Rate in AsiaFig. 8: Number of Novel Coronavirus (COVID-19) Cases of Selected Countries in the Asia Pacific Region as of

July 2, 2020

The Asian century is about to begin share of world GDP a PPP \$
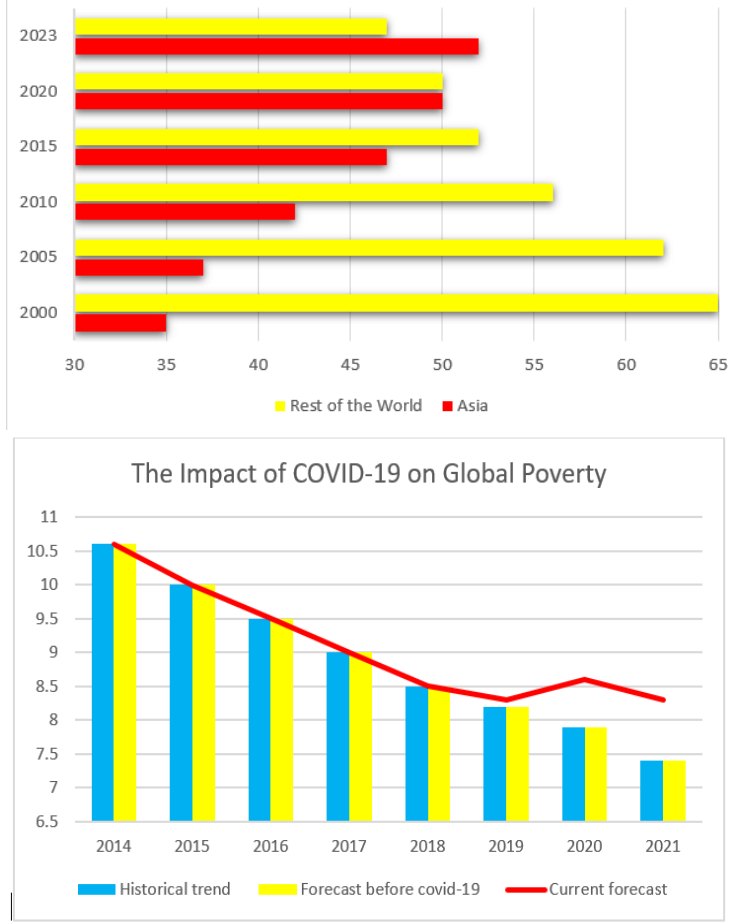

Fig. 9: GDP Status of AsiaFig. 10: Prediction on the Impact of COVID-19 on Global

Poverty

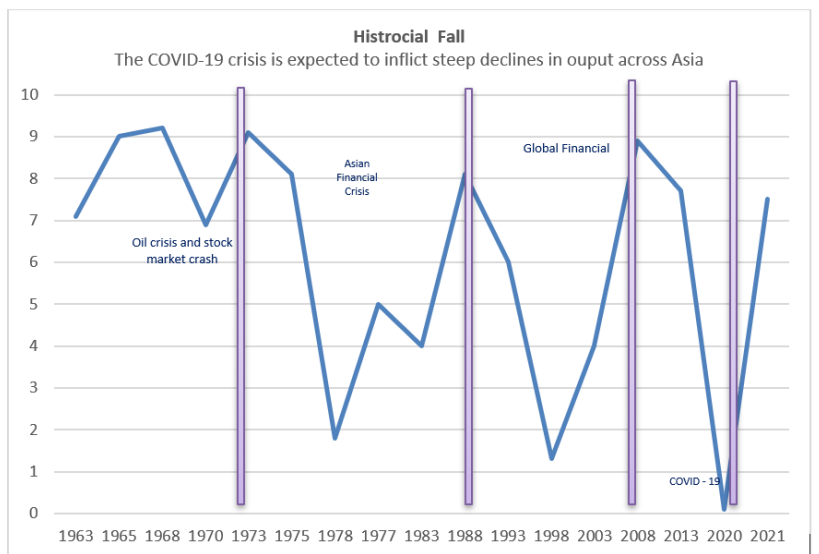

Fig. 11: Various Historical Falls in Asia

To offer assistance to analyze COVID-19, machine learning is being put to activity to utilize confront filters to recognize side effects for example the GDP status as shown in fig. 9. The

Copyright () Authors 
impact of COVID -19 on global poverty is predicted in fig.10. The prediction analysis also helps in analyzing various Asian crisis which happened in the past and also predicts that this COVID-19 which is depicted in fig.11.It is additionally coordinating with wearable innovation such as smart observes to hunt for tell-tale designs in a patient's resting heart rate, and machine learning-powered chatbots can screen patients based on self-reported symptoms [3-5].

\subsection{Personalized Medicine}

By applying $\mathrm{AI}$ and $\mathrm{ML}$ to numerous information sources-genetic information, electronic wellbeing records, sensor/wearables information, natural and way of life dataresearchers are taking to begin with steps toward creating personalized medicines for illnesses. This widespread modern need the medication world to speed up sedate advancement, come up quick with immunization, and a dependable symptomatic strategy. The current strategies being put to utilize include a parcel of trial and mistake, making them horrendously moderate. Whereas it can take months to separate one reasonable immunization candidate indeed, Machine learning can speed up this handle basically without compromising on quality control. Not fair unused drugs, the issue to calls for distinguishing compelling existing drugs. Machine learning can offer assistance here, as well. Restorative experts can prioritize sedate candidates much quicker by ML, consequently building information charts and anticipating intuitive between drugs and viral proteins.

\subsection{Drug Development}

ML can be connected at all stages of unused medicate revelation counting planning the chemical/protein structure of drugs, target approval, examining sedate security, and overseeing clinical trials. The trust is that utilizing
ML in medicate revelation will not as it was offer assistance altogether decrease the toll of presenting unused drugs to the showcase but to make the medicate disclosure handle quicker and more costeffective. AI company Atom Wise's stage Atom Net employments a profound learning computer program to filter through millions of conceivable particles in a day or two, which would regularly take months by means of conventional strategies. The program at that point analyzes reenactments that appear how the potential pharmaceutical will carry on within the human body. It has been able to recognize conceivable medications for different sclerosis and dangerous Ebola infection. Deep mind, the AI arm of Google's parent Letter set Inc, is additionally making tremendous advances in this field.

\subsection{Discover New Trends/Anomalies}

Machine learning is utilized when a computer has been instructed to recognize designs by giving it information and a calculation to get that information. This method of learning from the information is called training and the yield that we accomplish is tested. These strategies take after a set of rules utilizing endless sums of computing control. The models utilized in machine learning are classification, clustering, and regression. The reason for classification is to decide a name for the given information. Though clustering is utilized when an entire bunch of information is given but confirms result is not available which suggests that the given information is unlabeled. In this step, the unmistakable design is checked within the information.

\section{Other Applications of Machine Learning Health Care}

Machine learning-based robotic surgery is changing the way surgery is performed nowadays. The da Vinci robot is planned to encourage 
complex surgery employing a negligibly intrusive approach, decreasing the length of surgeries and along these lines' clinic remains. Different other mechanical apparatuses such as Stereotaxis in cardiac catheterization, Medtronic/Mazor in spine and neurology, Exactness in cancerous tumor light, Stryker's Mako in orthopedic hip and knee substitution are progressing surgical results for thousands of patients. Indeed, dental inserts and hair transplants are being performed by surgical robots nowadays. Machine learning has a widespread application as depicts in fig. 12 .

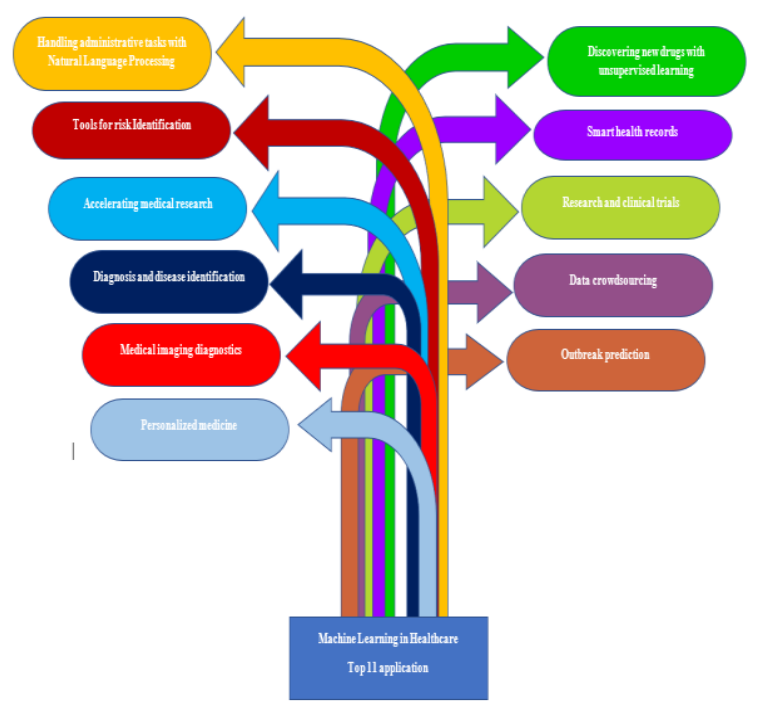

Fig. 12: Applications of Machine Learning in

\section{Medical Management}

AI and ML-based methods will improve the accuracy of surgical instruments by joining real-time information, criticism from past effective surgeries, and information from electronic therapeutic records amid the surgery itself. This mayoffer assistance which decreases human mistake and offers assistance to common specialists to perform complex surgeries in resource-limited settings.
TABLE 1: Existing Machine Learning

Software in Medical Management

\begin{tabular}{|c|c|c|}
\hline SNO & $\begin{array}{c}\text { NAME OF THE } \\
\text { SOFTWARE }\end{array}$ & DESCRIPTION \\
\hline 1 & $\begin{array}{l}\text { QUOTIENT } \\
\text { HEALTH }\end{array}$ & $\begin{array}{l}\text { With this learning } \\
\text { application, wellbeing } \\
\text { segments are able to } \\
\text { diminish their } \\
\text { wellbeing care costs } \\
\text { since it was } \\
\text { particularly outlined } \\
\text { to assist in lessening } \\
\text { the taken a toll of } \\
\text { supporting electronic } \\
\text { restorative records } \\
\text { systems }\end{array}$ \\
\hline 2 & $\begin{array}{l}\text { KENSCI } \\
\text { LEARNING } \\
\text { MACHINE }\end{array}$ & $\begin{array}{l}\text { This machine } \\
\text { learning in healthcare } \\
\text { application is being } \\
\text { utilized for } \\
\text { determination } \\
\text { purposes. That's, it } \\
\text { makes a difference } \\
\text { specialist identify any } \\
\text { shape of sickness in } \\
\text { their patient's body, } \\
\text { that will happen } \\
\text { within the closest } \\
\text { future. And through } \\
\text { this, doctors are } \\
\text { regularly able to } \\
\text { endorse preventive } \\
\text { drugs that will } \\
\text { annihilate such } \\
\text { sickness sometime } \\
\text { recently it develops. }\end{array}$ \\
\hline 3 & $\begin{array}{l}\text { CIOX HEALTH } \\
\text { MACHINE }\end{array}$ & $\begin{array}{lr}\text { This } & \text { healthcare } \\
\text { application } & \text { makes } \\
\text { difference } & \text { support. }\end{array}$ \\
\hline
\end{tabular}




\begin{tabular}{|c|c|c|}
\hline & & $\begin{array}{l}\text { The spread of health } \\
\text { information from one } \\
\text { office to the other is } \\
\text { done through this. }\end{array}$ \\
\hline 4 & PATHAI & $\begin{array}{l}\text { pathologist to } \\
\text { immediately } \\
\text { recognize sickness in } \\
\text { their limit, and after } \\
\text { that choose whether } \\
\text { or not such patients } \\
\text { would require an } \\
\text { unused shape of } \\
\text { treatment or proceed } \\
\text { to utilize medicate } \\
\text { treatment. }\end{array}$ \\
\hline 5 & $\begin{array}{l}\text { QUANTITATIVE } \\
\text { INSIGHTS }\end{array}$ & $\begin{array}{l}\text { With its MRI } \\
\text { workstation Quantx, } \\
\text { this machine rapidly } \\
\text { spots out breast } \\
\text { cancer most } \\
\text { particularly. It is one } \\
\text { of the foremost well- } \\
\text { known applications } \\
\text { utilized in treating } \\
\text { cancer }\end{array}$ \\
\hline 6 & $\begin{array}{l}\text { INNEREYE } \\
\text { (MICROSOFT) }\end{array}$ & $\begin{array}{l}\text { This application } \\
\text { makes doctors } \\
\text { recognize between } \\
\text { solid life systems and } \\
\text { tumors, through its } \\
\text { 3D radiological } \\
\text { shown photographs. } \\
\text { Also, it moreover } \\
\text { makes } \\
\text { specialistarrange their } \\
\text { surgical operations } \\
\text { precisely }\end{array}$ \\
\hline 7 & PFIZER & $\begin{array}{l}\text { This machine, } \\
\text { through the assistance } \\
\text { of IBM's Watson AI }\end{array}$ \\
\hline
\end{tabular}

\begin{tabular}{|l|l|}
\hline & $\begin{array}{l}\text { innovation, makes } \\
\text { doctors by giving } \\
\text { thoughts to how one's } \\
\text { body safe framework } \\
\text { can battle off cancer }\end{array}$ \\
\hline
\end{tabular}

\section{Appropriateness of Machine Learning in Medical Management}

Machine learning, basically, could be a sort of artificial intelligence when computers are modified to memorize data without human mediation. In machine learning, the improvement of the underlying algorithms depends on computational insights. Computers are given information and after that, the computers "learn" from that information. The information really "teaches" the computer by uncovering its complex designs and fundamental calculations. The bigger the test of information the "machine" is given, the more exact the machine's yield gets to be. Few existing machine learning applications for medical management are depicted in Table 1.

Machine learning in healthcare is getting to be more broadly utilized and is making different patients and clinicians in numerous diverse ways. The foremost common healthcare utilizes cases for machine learning are robotizing therapeutic charging, clinical choice bolster, and the advancement of clinical care rules. There are numerous eminent illustrations of machine learning and healthcare concepts being connected in medication. At MD Anderson, researchers have created the primary therapeutic machine learning calculation to anticipate intense toxicities in patients getting radiation treatment for head and neck cancers. Google's machine learning applications in healthcare were prepared to identify breast cancer and accomplished 89 percent exactness, on standard or superior to radiologists. 
These are fair to some of the cases of the numerous employments of machine learning in healthcare.

Unstructured healthcare information for machine learning grows to nearly $80 \%$ of the data held or "locked" in electronic wellbeing record frameworks. These are not information components but reports or content records which within the past seem not to be analyzed without a human perusing through the fabric. Human dialect, or "natural language," is exceptionally complex, missing consistency, and consolidates a colossal sum of equivocalness, language, and unclearness. In order to change over these reports into more valuable and analyzable information, machine learning in healthcare frequently depends on NLP programs. Most profound learning in healthcare applications that utilize NLP requires a few frames of restorative machine learning.

Medical management utilizes machine learning in numerous ways. For case, the same NLP innovation that's utilized to decide financial soundness for a shopper or estimation investigation of someone's social media post can presently be utilized to perused a patient's chart to extricate important data elements just like the patient's drugs, treatment plans, and restorative conditions.

Machine learning therapeutic information comprises preparing our machines to analyze the discourse designs of our doctor's conclusion clients and decide the setting of vital therapeutic terms. Our strong refutation motor can recognize not as it were key terms, but moreover, all four invalidation sorts: theoretical,negative, history, and family history are the four vital invalidation sorts. With over 500 refutation terms machine is able to attain exactness rates that are more prominent than $97 \%$.

But not everything is done by the machine. Healthcare information for machine learning must be arranged in such a way that the computer can more easily discover designs and inductions. Typically as a rule done by people that tag components of the dataset as a comment over the input. Our group of clinical specialists is performing this work as well as analyzing comes about, composing unused rules, and improving machine execution. Be that as it may, in arrange for the machine learning applications in healthcare to memorize productively and viably, the comment done on the information must be precise, and significant to the errand of extricating key concepts with the appropriate setting.

\section{Concerns}

One must keep in mind that amazing specialized developments in AI cannot settle social/political issues. Moreover, the information input to $\mathrm{AI}$ must be in large volume and of clinically large quality/relevance. In a general sense, imperfect information cannot substitute for huge volumes. Currently, most of the AI applications are utilizing the worldview of 'deductive reasoning' and haveto move towards 'inductive reasoning'.

Cybersecurity and protection are major concerns in healthcare, particularly ensuring quiet security. In spite of the fact that steps have been taken to prioritize security, machine learning might offer assistance to decrease any potential dangers through the era of clever calculations and solid security of clinical wellbeing information. Machine learning apparatuses could be able to recognize EHR utilization designs and thus distinguish any extraordinary occasions at a quicker rate than people can, hence changing specialists and lessening the chance of security breaches.

\section{CONCLUSION}

Advancements like personalized medications and independent mechanical surgery are gradually picking up energy as the healthcare division is leveraging Machine Learning to its best. 
Suggestions on medical management and medications for maladies are personalized based on the patient's therapeutic history, slim down, stretch levels, past conditions, etc. Robots are being prepared to perform complicated surgeries on people which, as well, as effective as a human specialist. Envision a period where a persistent can filter a picture of his medical status through his phone and know what kind of malady he is enduring with. In a nutshell, machine learning in healthcare has colossally raised the standard of the therapeutic organization from the winding, timeconsuming mode of operation, to a basic, faster, and more exact bureaucracy. As clinics consider joining $\mathrm{AI}$ and machine learning into their budgets and procedures, numerous questions emerge when considering approximately the effect of this unused innovation on a large scale for big data.

\section{DECLARATION OF INTERESTS}

The authors declare that they have no known competing financial interests or personal relationships that could have appeared to influence the work reported in this paper.

\section{REFERENCES:}

$$
\text { COVID-19 }
$$

Analytics,

http://www.covidanalytics.io/2. [online- accessed date $7 / 7 / 2020$ ]

[2] Coronavirus - $\quad$ worldometer,

link: https://www.worldometers.info/coronavirus/, [online- accessed date 7/7/2020]

[3] G. Li, E. De Clercq, Therapeutic options for the 2019 novel coronavirus (2019-ncov), 2020.

[4] S. MallapatyWhat the cruise-ship outbreaks reveal about COVID-19

Nature, 580 (7801) (2020) 18-18

$$
\text { K. Liu, Y. Chen, R. Lin, K. HanClinical }
$$
features of COVID-19 in elderly patients: a comparison with young and middle-aged patients J. Infect. (2020)
[6]S. Zhao, Q. Lin, J. Ran, S.S. Musa, G. Yang, W. Wang, Y. Lou, D. Gao, L. Yang, D. He, et

al.Preliminary estimation of the basic reproduction number of novel coronavirus (2019-ncov) in china, from 2019 to 2020: adata-driven analysis in the early phase of the outbreak Int. J. Infect. Dis., 92 (2020), pp. 214-217

[7] S. Tuli, S. Tuli, G. Wander, P. Wander, S.S. Gill, S. Dustdar, R. Sakellariou, O. Rana, Next generation technologies for smart healthcare: challenges, vision, model, trends and future directions, Internet Technol. Lett.e145.

[8]C. Huang, Y. Wang, X. Li, L. Ren, J. Zhao, Y. Hu, L. Zhang, G. Fan, J. Xu, X. Gu, et al. Clinical features of patients infected with 2019 novel coronavirus in Wuhan, China The Lancet, 395 (10223) (2020), pp. 497-506

[9]A. Depeursinge, A.S. Chin, A.N. Leung, D. Terr one, M. Bristow, G. Rosen, D.L. RubinAutomated classification of usual interstitial pneumonia using regional volumetric texture analysis in highresolution CTInvest. Radiol., 50 (4) (2015), p. 261 [10] Jin, B. Wang, H. Xu, C. Luo, L. Wei, W. Zhao , X. Hou, W. Ma, Z. Xu, Z. Zheng, et al.AI-assisted CT imaging analysis for covid-19 screening: building and deploying a medical ai system in four weeks medRxiv (2020).

[11] M.W. Libbrecht, W.S. NobleMachine learning applications in genetics and genomics Nat. Rev. Genet., 16 (6) (2015), pp. 321-332

[12]E. Chen, K. Lerman, E. Ferrara, COVID-19: The First Public Coronavirus Twitter Dataset, arXiv preprint: 1603.07252(2020).

[13]There's an unlikely beneficiary of coronavirus: the planets, link: https:/edition.cnn.com/2020/03/16/asia/chinapollution-coronavirus-hnk-intl/index.html, [online accessed].

[14]. Milinovich GJ, Williams GM, Clements $\mathrm{AC}$, et al. Internet-based surveillance systems 
for monitoring emerging infec-tious diseases [ J] . The Lancet Infectious Diseases, 2014( 14):160-168.

[15]. Huang P. Research and Implementation of Prediction Model for Class B Infectious Diseases Based on Machine Learning [D]. University of Electronic Science and Technology, 2019.

[16]. Li J, Lu L, Wu J, et al. Application of Feedback Neural Network Model in Infectious Disease Prediction $[\mathrm{J}]$. Chinese Journal of Preventive Medicine, 2011(07):75-78.

[17]. Han B A, Paul S J, Alexander L W, et al. Undiscovered Bat Hosts of Filoviruses[J]. PLOS Neglected Tropical Diseases, 2016, 10(7)

[18]. Rong Z. Design and validation of Ebola epidemic disease prediction model and prevention and control strategy based on STEM platform [D]. Northwestern University, 2019.

[19].https://baike.baidu.com/item/SARS\%E4\%BA $\% 8 \mathrm{~B} \%$ E4\%BB\%B6/7702261\#reference-[7]1996352-wrap

[20]. Yang Z F, Zeng Z Q,Wang K et al., Modified SEIR and AI prediction of the epidemics trend of COVID-19 in China under public health interventions. 2020

[21]

C. Wang, P.W. Horby, F.G. Hayden, G.F. GaoA novel coronavirus outbreak of global health concernThe Lancet, 395 (10223) (2020), pp. 470473 